\title{
The level of proinflammatory cytokines: interleukins 12, 23, 17 and tumor necrosis factor $\alpha$ in patients with metabolic syndrome accompanying severe psoriasis and psoriatic arthritis
}

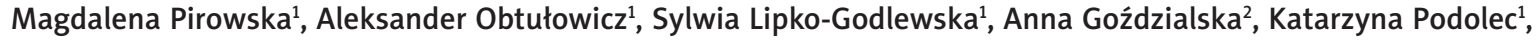 \\ Anna Wojas-Pelc ${ }^{1}$ \\ ${ }^{1}$ Department of Dermatology, Jagiellonian University Medical College, Krakow, Poland \\ ${ }^{2}$ Faculty of Medicine and Health Sciences, Andrzej Frycz Modrzewski Krakow University, Krakow, Poland \\ Adv Dermatol Allergol 2018; XXXV (4): 360-366 \\ DOI: https://doi.org/10.5114/ada.2018.77665
}

\begin{abstract}
Introduction: The incidence of metabolic syndrome is estimated at $15-24 \%$ in the general population and at $30-50 \%$ in patients with psoriasis. A probable cause of the described correlation is a constant release in chronic dermatosis of proinflammatory cytokines and their influence on individual systems and organs.

Aim: Assessment of the concentration of the proinflammatory cytokines (IL-12, IL-23, IL-17 and TNF- $\alpha$ ) in blood serum and their correlation with the intensity of skin lesions, the presence of psoriatic arthritis and the risk of development of obesity and metabolic syndrome.

Material and methods: The concentrations of subunit p70 IL-12, IL-17 and IL-23, and TNF- $\alpha$ in subjects with psoriasis and metabolic syndrome were determined.

Results: The level of the studied cytokines, IL-17, IL-23 and TNF- $\alpha$ was higher in patients diagnosed with psoriasis. Higher levels of IL-17, IL-23 and TNF- $\alpha$ were observed in patients with metabolic syndrome accompanying psoriasis. A higher level of IL-17 and IL-23 was found in sera of patients with psoriatic arthritis in comparison to normal psoriasis.

Conclusions: In the study, a higher level of IL-17 and IL-23 was also shown in patients with psoriatic arthritis in comparison to patients with normal psoriasis. The effectiveness of anti-IL12/23 drugs in psoriatic arthritis is a confirmation of the obtained results of the studies. Additionally, the increased level of IL-17, both in patients with metabolic syndrome and with psoriasis, could indirectly indicate an increased cardiovascular risk in patients with affected joints in comparison to psoriasis affecting only the skin.
\end{abstract}

Key words: psoriasis, metabolic syndrome, cytokines.

\section{Introduction}

Psoriasis (PsO) is a chronic inflammatory disease with immunologic etiology, depending mainly on T lymphocytes and dendritic cells [1]. Many previous studies have demonstrated a more frequent coincidence of dermatosis with other systemic diseases, such as metabolic syndrome (MS), cancers, ischemic heart disease, chronic obstructive pulmonary disease, inflammatory bowel diseases, depression, and osteoporosis [2-4]. The incidence of metabolic syndrome is estimated at $15-24 \%$ in the general population and at 30-50\% in patients with psoriasis [5]. A probable cause of the described correlation is a constant release in chronic dermatosis, over the years, of proinflammatory cytokines, mainly tumor necrosis factor $\alpha$ (TNF- $\alpha$ ) and interleukin (IL)-6, and their influence on individual systems and organs [6]. It is known that mainly IL-12 and IL-23, released by dendritic cells, are important in the immune etiology of psoriasis. They activate Th1 and Th2 to produce many cytokines: IL-17, interferon (IFN)- $\gamma$, TNF- $\alpha$ and IL-22, which, by direct stimulation of keratinocytes, cause the formation of psoriatic lesions [1]. The described etiology is confirmed by the results of therapies with antibodies against the above inflammatory molecules, commonly used in the psoriasis treatment. The results of previous studies unambiguously

Address for correspondence: Magdalena Pirowska MD, PhD, Department of Dermatology, Jagiellonian University Medical College, 8 Skawinska St, 30-066 Krakow, Poland, phone: +48 600858 891, e-mail: pirowska@gmail.com Received: 24.02.2017, accepted: 24.05.2017. 
confirm the correlation between the TNF- $\alpha$ in serum and the intensity of skin lesions. The results of studies on the concentration of IL-17 are IL-23 are ambiguous - some of them indicate a strong correlation between the severity of the disease and the level of circulating interleukins, however, there are reports where the evaluated cytokines were indeterminable despite the advancement of the disease [7].

In recent years, the influence of methotrexate on the reduction of the risk of cardiovascular diseases has been described many times. The mechanism of this phenomenon is not known [8]. Currently, the studies are conducted to explain the influence of biological drugs on the development of the individual components of the metabolic syndrome. There are many reports on the reduction of insulin resistance, hyperlipidemia and waist circumference in patients receiving anti-TNF $[9,10]$. However, experimental studies on mice showed that IL-17 can be a negative regulator of adipogenesis and glucose level and hence it delays the development of obesity [11]. Subsequent studies are necessary to indicate the profile of inflammatory cytokines in patients with psoriasis accompanied by metabolic syndrome, in order to evaluate the potential influence of biological drugs on the reduction of the incidence of complications in the form of diabetes, ischemic heart disease, hypertension or hypercholesterolemia.

\section{Aim}

Considering the most widely described influence of IL-12, IL-23, IL-17 and TNF- $\alpha$ on the formation of psoriatic plaques, an attempt was made to assess the concentration of the above proinflammatory cytokines in blood serum and their correlation with the intensity of skin lesions, the presence of psoriatic arthritis and the risk of development of obesity and metabolic syndrome. The association between levels of cytokines in psoriasis and presence of metabolic syndrome could imply the treatment, especially therapy that is correlated with IL-17, IL-23 or TNF- $\alpha$.

\section{Material and methods}

For this study, 60 patients over 18 years of age were included (24 females and 36 males), diagnosed and treated for severe psoriasis (PASI, BSA > 10) in the Dermatology Clinic of the Jagiellonian University Medical College. Control groups comprised 15 subjects diagnosed with metabolic syndrome and 15 healthy voluntary subjects. In both groups, the exclusion criteria were acute and chronic inflammatory diseases with the exception of diabetes, hypertension and dyslipidemia.

For testing, venous blood ( $5 \mathrm{ml}$ ) was collected after the night rest. The concentrations of subunit p70 IL-12, IL-17 and IL-23 and TNF- $\alpha$ in subjects' sera were deter- mined by Quantikine test with enhanced sensitivity, with the ELISA method. The expected values were: IL-12 ND - 3.58 pg/ml; IL-17 below 31.2 pg/ml; IL-23 ND - 40.5 pg/ $\mathrm{ml}$; and TNF- $\alpha 0.550-2.816 \mathrm{pg} / \mathrm{ml}$. The advancement of psoriasis was evaluated with PASI and BSA scales. The PASI scale (psoriasis area and severity index) combines three physical features: (erythema, desquamation and induration), as three separate parameters, calculated for every part of the body separately, considering their intensity and the percentage of the affected area. Psoriatic arthritis was diagnosed based on the Caspar criteria (2006). Metabolic syndrome was diagnosed based on the presence of revised NCEP-ATP III criteria for metabolic syndrome, according to ANA/NHBLI.

A multivariable logistic regression analysis was performed to identify the factors that were independently associated with levels of II-17, IL-23 and TNF- $\alpha$. Data were summarized descriptively and differences in dichotomous and continuous variables were examined between patients with and without psoriasis and metabolic syndrome. All data were analyzed by Statistica using MannWhitney and Spearman's rho. A $p$-value $\leq 0.05$ was considered significant.

For the above studies, consent of the Bioethics Committee of the Jagiellonian University was obtained.

\section{Results}

The IL-12 level in all the subjects was indeterminable $(<0.78 \mathrm{pg} / \mathrm{ml})$. The level of IL-23 and TNF- $\alpha$ was higher in patients diagnosed with psoriasis in comparison to the control group (serum concentration mean values of IL-23 were: $8.33 \pm 7.83$ vs. $3.13 \pm 3.11(p<0.05)$ and TNF- $\alpha 1.98$ \pm 1.50 vs. $1.13 \pm 0.42(p<0.05)$. The concentration of IL-17 in the study group $(2.56 \pm 3.15)$, compared to the control group $(2.07 \pm 4.23)$ was higher, however the results were not statistically significant ( $p=0.52$ ) (Figure 1 , Table 1 ). Higher levels of IL-17, IL-23 and TNF- $\alpha$ were observed in patients with metabolic syndrome accompanying psoriasis in comparison to patients with chronic dermatosis without the features of this syndrome, however they were significantly different only between the IL-17 groups: IL-17 4.05 vs. 2.09; $p<0.05$; IL-23 8.12 vs. 8.25; $p=0.41$; TNF- $\alpha 2.42$ vs. 1.83; $p=0.42$ (Figure 2, Table 2). There was no significant change in the serum concentration of all cytokines in the control group in patients with metabolic syndrome in comparison to subjects without the syndrome (TNF- $\alpha 1.21 \pm 0.43$; vs. $0.94 \pm 1.43$; $p=0.14$; IL-23 $2.77 \pm 1.85$ Vs. $4.52 \pm 4.76 ; p=0.19 ;$ IL-17 $2.20 \pm 1.55$ vs. $2.03 \pm 1.57 ; p=0.77)$. No positive correlation was found between PASI, BSA and the level of cytokines (IL-17, IL-23, TNF- $\alpha$ ).

Among the patients with psoriasis and psoriatic arthritis, no correlation was observed between the level of IL-17, IL-23, TNF- $\alpha$ and BMI $(r=0.03, p=0.79 ; r=-0.13$, $p=0.32 ; r=0.10, p=0.44)$. The observations between 

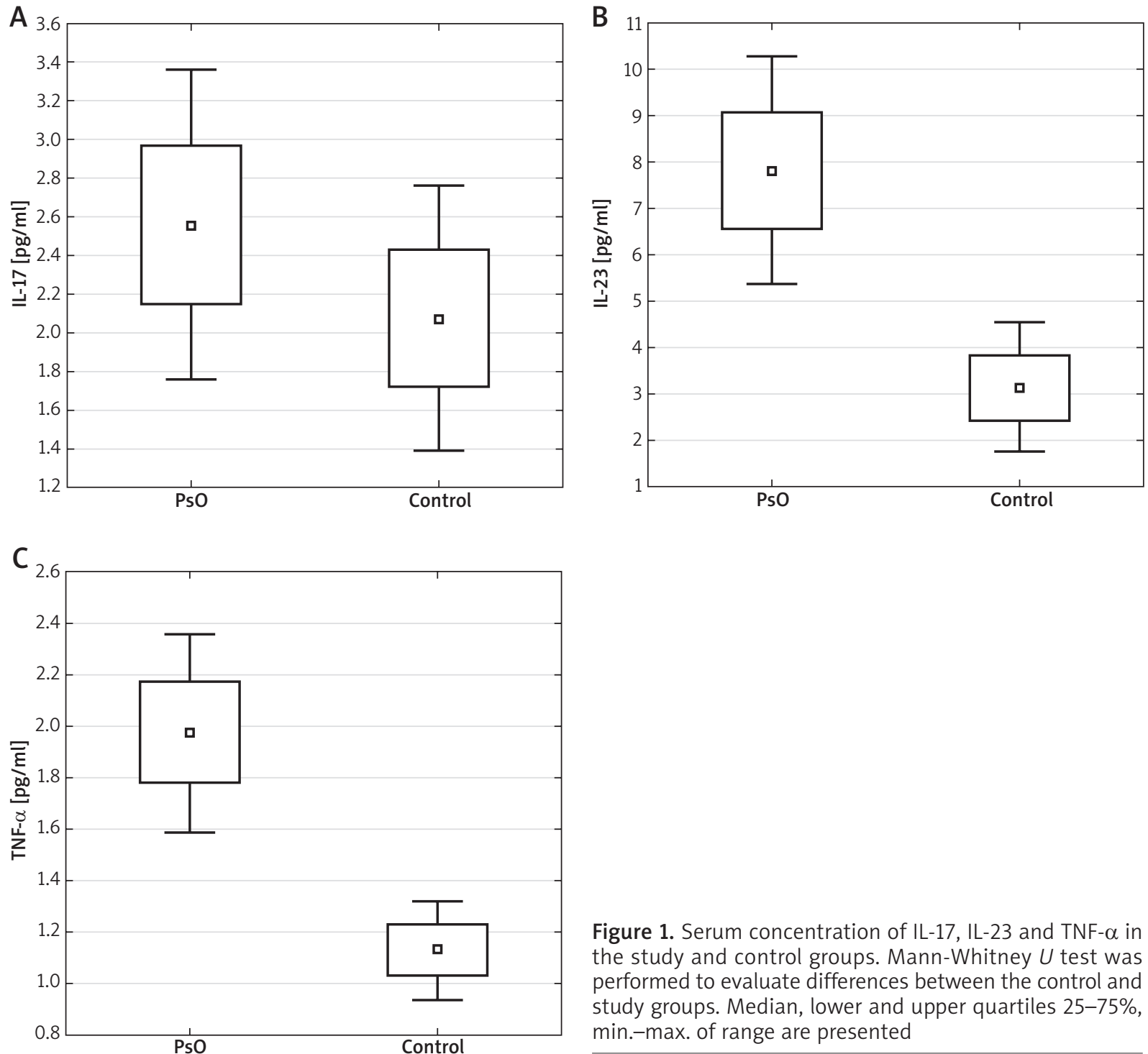

Figure 1. Serum concentration of IL-17, IL-23 and TNF- $\alpha$ in the study and control groups. Mann-Whitney $U$ test was performed to evaluate differences between the control and study groups. Median, lower and upper quartiles $25-75 \%$, min.-max. of range are presented

Table 1. Serum concentration of IL-17, IL-23 and TNF- $\alpha$ : detailed results in the psoriasis group and the control group

\begin{tabular}{|c|c|c|c|c|c|c|}
\hline \multirow[t]{2}{*}{ Parameter } & \multicolumn{2}{|c|}{ IL-17 assay results [pg/ml] } & \multicolumn{2}{|c|}{ IL-23 assay results $[\mathrm{pg} / \mathrm{ml}]$} & \multicolumn{2}{|c|}{ TNF- $\alpha$ assay results $[\mathrm{pg} / \mathrm{ml}]$} \\
\hline & PsO & Control group & PsO & Control group & PsO & Control group \\
\hline Valid observations & 60 & 30 & 60 & 30 & 60 & 30 \\
\hline Mean value & 2.56 & 2.07 & 8.33 & 3.13 & 1.98 & 1.13 \\
\hline Median & 1.88 & 1.56 & 8.12 & 3.01 & 1.77 & 1.02 \\
\hline Minimum value & 1.75 & 1.39 & 5.36 & 4.66 & 1.59 & 0.97 \\
\hline Maximum value & 3.37 & 2.77 & 10.31 & 1.77 & 2.36 & 1.33 \\
\hline Lower quartile & 2.13 & 1.70 & 6.61 & 2.42 & 1.79 & 1.04 \\
\hline Upper quartile & 2.98 & 2.42 & 9.11 & 3.88 & 2.18 & 1.22 \\
\hline Standard deviation & 3.15 & 4.23 & 7.83 & 3.11 & 1.50 & 0.42 \\
\hline $\begin{array}{l}\text { Statistical significance } \\
\text { (Mann-Whitney } U \text { test) }\end{array}$ & \multicolumn{2}{|c|}{$p=0.52$} & \multicolumn{2}{|c|}{$p=0.0011$} & \multicolumn{2}{|c|}{$p=0.032$} \\
\hline
\end{tabular}



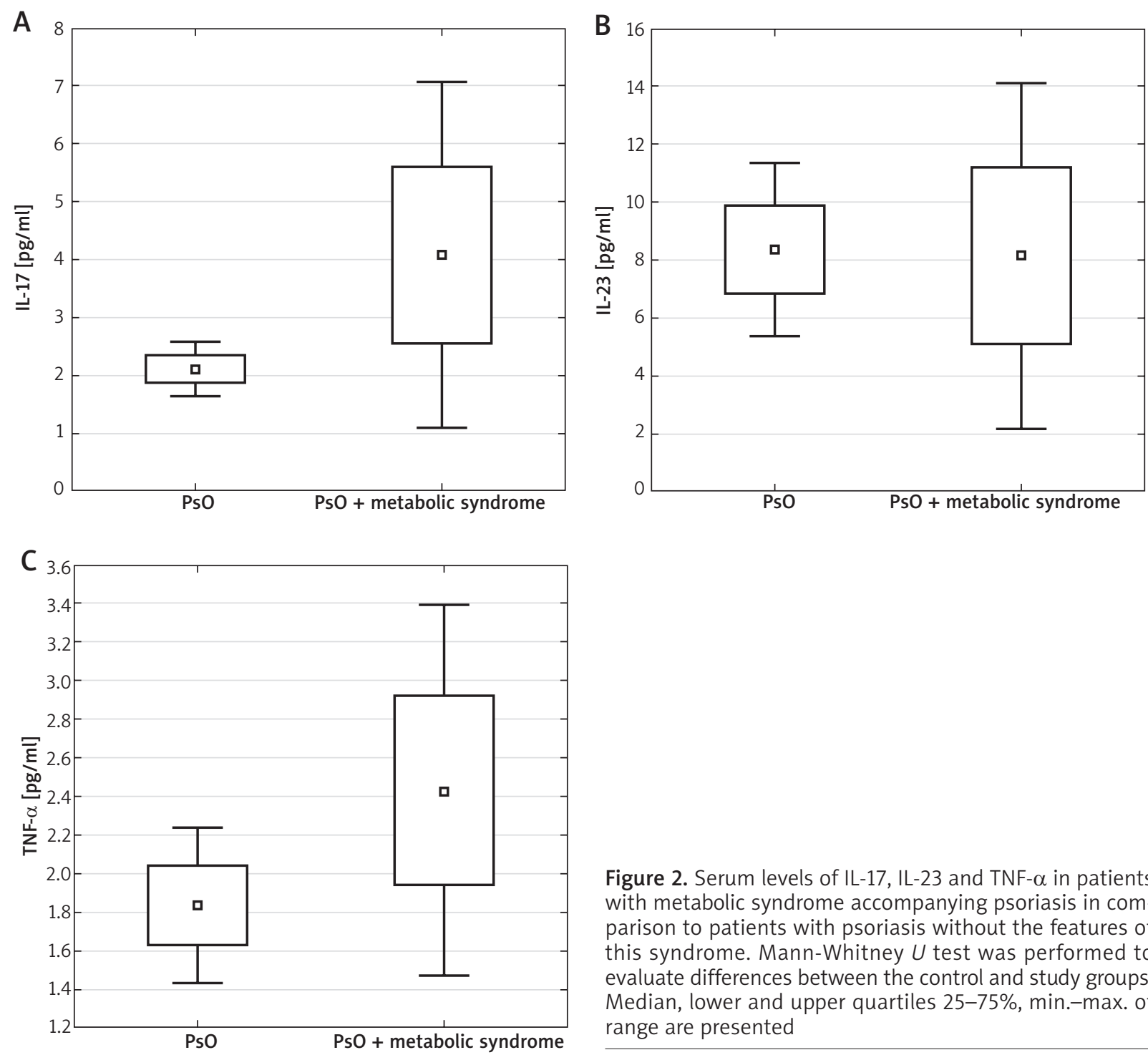

Figure 2. Serum levels of IL-17, IL-23 and TNF- $\alpha$ in patients with metabolic syndrome accompanying psoriasis in comparison to patients with psoriasis without the features of this syndrome. Mann-Whitney $U$ test was performed to evaluate differences between the control and study groups. Median, lower and upper quartiles $25-75 \%$, min.-max. of range are presented

Table 2. Serum levels of IL-17, IL-23 and TNF- $\alpha$ : detailed results in patients with metabolic syndrome accompanying psoriasis in comparison to patients with psoriasis without the features of this syndrome

\begin{tabular}{|c|c|c|c|c|c|c|}
\hline \multirow[t]{2}{*}{ Parameter } & \multicolumn{2}{|c|}{ IL-17 assay results [pg/ml] } & \multicolumn{2}{|c|}{ IL-23 assay results [pg/ml] } & \multicolumn{2}{|c|}{ TNF- $\alpha$ assay results $[\mathrm{pg} / \mathrm{ml}]$} \\
\hline & $P s+M S$ & Ps/no MS & $\mathrm{Ps}+\mathrm{MS}$ & Ps/no MS & $\mathrm{Ps}+\mathrm{MS}$ & Ps/no MS \\
\hline Valid observations & 30 & 30 & 30 & 30 & 30 & 30 \\
\hline Mean value & 4.05 & 2.09 & 8.12 & 8.25 & 2.42 & 1.83 \\
\hline Median & 3.65 & 1.86 & 5.12 & 6.37 & 1.89 & 1.13 \\
\hline Minimum value & 1.10 & 1.71 & 2.10 & 5.25 & 1.44 & 1.47 \\
\hline Maximum value & 7.03 & 2.61 & 14.02 & 11.41 & 2.93 & 3.40 \\
\hline Lower quartile & 2.58 & 1.88 & 5.07 & 6.90 & 1.92 & 1.93 \\
\hline Upper quartile & 5.62 & 2.36 & 11.29 & 9.94 & 2.92 & 1.92 \\
\hline $\begin{array}{l}\text { Statistical significance } \\
\text { (Mann-Whitney } U \text { test) }\end{array}$ & \multicolumn{2}{|c|}{$p=0.0001$} & \multicolumn{2}{|c|}{$p=0.41$} & \multicolumn{2}{|c|}{$p=0.42$} \\
\hline
\end{tabular}



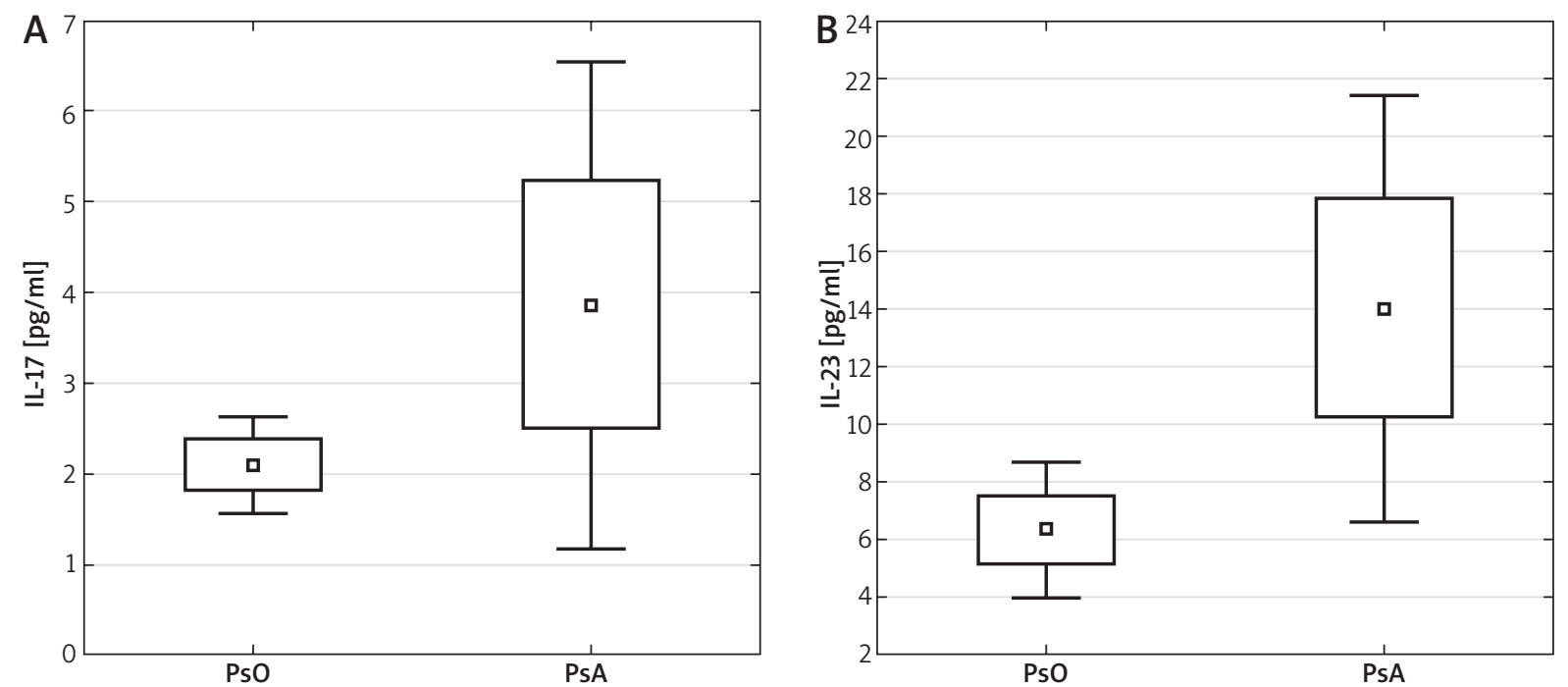

Figure 3. Level of IL-17 and IL-23 in sera of patients with psoriatic arthritis (PsA) in comparison to psoriasis (PsO). MannWhitney $U$ test was performed to evaluate differences between the control and study groups. Median, lower and upper quartiles $25-75 \%$, min.-max. of range are presented

the waist circumference and cytokines were similar: TNF- $\alpha(r=0.15, p=0.26)$ and IL-17 $(r=0.09, p=0.50)$ IL-23 $(r=-0.06, p=0.66)$. Among the patients with psoriasis and psoriatic arthritis, no correlation was observed between PASI and the body mass, waist circumference and BMI.

A higher level of IL-23 was found in sera of patients with psoriatic arthritis in comparison to normal psoriasis (PsO $6.35 \pm 7.89$ vs. PsA $14.03 \pm 14.63$; $p<0.05$ ), no positive correlation of IL-17 in these groups was observed (PsO $2.11 \pm 1.82$ vs. PsA $3.88 \pm 5.38 ; p=0.059$ ) (Figure 3, Table 3). Among the patients with psoriatic arthritis, no higher incidence of overweight and obesity was observed than for patients with normal psoriasis (BMI $27.7 \pm 5.68$ vs. $28.1 \pm 5.22 \mathrm{~kg} / \mathrm{m}^{2}$ ).

\section{Discussion}

Previous studies show that in the pathogenesis of psoriasis, a special role is played by subpopulations of Th lymphocytes and dendritic cells together with cytokines produced by them: Th1 produce IFN- $\gamma$ and IL-4 and IL-10, Th17 - IL-17 and IL-22, while Th22 - IL-22 and cytotoxic epithelial lymphocytes T CD8+ [12]. On the other hand, the Th17/IL-23 axis is crucial in maintaining the chronic inflammation, characteristic of the described dermatosis [13]. Interlekin-23, produced by dendritic cells, is a key cytokine taking part in autoimmunisation as it induces reproduction and maturation of Th17 cells. The studies performed confirmed increased levels of IL-23 and TNF- $\alpha$ in the blood of patients with psoriasis in comparison to the control group.

Table 3. Levels of IL-17 and IL-23: detailed results of patients with psoriatic arthritis (PsA) in comparison to psoriasis (PsO)

\begin{tabular}{|c|c|c|c|c|}
\hline \multirow[t]{2}{*}{ Parameter } & \multicolumn{2}{|c|}{ IL-17 assay results [pg/ml] } & \multicolumn{2}{|c|}{ IL-23 assay results $[\mathrm{pg} / \mathrm{ml}]$} \\
\hline & PsO & PsA & PsO & PsA \\
\hline Valid observations & 26 & 34 & 26 & 34 \\
\hline Mean value & 2.11 & 3.88 & 6.35 & 14.03 \\
\hline Median & 1.88 & 2.73 & 2.12 & 4.31 \\
\hline Minimum value & 1.55 & 1.19 & 4.00 & 6.62 \\
\hline Maximum value & 2.65 & 6.58 & 8.89 & 21.36 \\
\hline Lower quartile & 1.84 & 2.50 & 5.19 & 10.19 \\
\hline Upper quartile & 2.41 & 5.28 & 7.36 & 19.91 \\
\hline Standard deviation & 1.82 & 5.38 & 7.89 & 14.63 \\
\hline Statistical significance (Mann-Whitney $U$ test) & \multicolumn{2}{|c|}{$p=0.059$} & \multicolumn{2}{|c|}{$p=0.0038$} \\
\hline
\end{tabular}


It is assumed that in the pathogenesis of the atherosclerotic plaque, a role is played by many cytokines: TNF- $\alpha$, IL-1, IL-6, IL-18, IL-33, IL-37, and also, important in the course of psoriasis, IL-12, IL-23 and IL-17 [14]. In the recent scientific reports, the prevailing theory has been the generation of the atherosclerotic plaque by chronic inflammation in the course of diseases such as rheumatoid arthritis or psoriasis. In the results obtained in the study, the patients with metabolic syndrome accompanying psoriasis had a higher level of studied cytokines than patients with the dermatosis only. The described results could have an important influence on the choice of the early method for patient treatment. Current reports point out the reduction of the risk of cardiovascular diseases and the reduction in glycemia and insulin resistance in patients treated with TNF- $\alpha$ inhibitors [15-17]. However, there are also studies showing an increase in the incidence of heart insufficiency and lipid disturbances in a similar group of patients $[18,19]$. Similarly inconsistent reports refer to the anti-IL-12/23 therapy [20]. However, there are no studies on the influence of anti-IL-17 drugs on the risk of development of the features of metabolic syndrome and cardiovascular diseases [16]. In the obtained results, the level of IL-17 was statistically significantly higher in the group of patients with metabolic syndrome accompanying psoriasis, which could indicate the influence of drugs from the anti-IL17 group not only on the dermatosis, but also on metabolic disturbances and cardiovascular incidents. The results seem especially significant in the context of the control group, where no increased level of IL-17 was found in patients with metabolic syndrome. Maybe IL-17 has a special influence on the occurrence of features of metabolic syndrome specifically in patients with psoriasis.

In the performed study, only a weak correlation of the intensity of the disease with the concentration of TNF- $\alpha$ was confirmed, not significant statistically. Similar results were obtained by other authors [21, 22]. However, there are reports confirming a strong correlation between the intensity of the disease and TNF- $\alpha$ [23]. No correlation was shown between IL-17 and PASI. Similar results of studies, referring to the same cytokines, were obtained by Kyriakou et al. and by El-Moaty-Zaher et al. [24]. On the other hand, Michalak-Stoma et al. and Arican et al. confirmed a positive correlation between the intensity of psoriasis and the level of IL-17 [25, 26].

It is assumed that obesity is related to persisting, chronic inflammation of a low degree [27].

In the studied group of patients, no strong positive correlation was observed between the level of IL-17 and TNF- $\alpha$ and the obesity expressed as the BMI or waist circumference. The relation between the anti-TNF- $\alpha$ treatment and the obesity is unclear. There are many works referring to the increase in body mass during the therapy [28-30]. Studies on mice showed that the deficiency of IL-17 increases the risk of diet-related obesity and accel- erates the accumulation of fat tissue even in animals on low-fat diet [11]. Giovanni et al. proved an indirect influence of IL-17 on the presence of early atherosclerosis in obese patients [31]. A negative correlation was observed between the level of IL-23 and the obesity. Similar results were obtained by Owczarczyk-Saczonek et al., in the group of patients treated with anti-IL12/23, studied by them, an increase in body mass and BMI was found during the therapy [32, 33].

In the study, a higher level of IL-17 and IL-23 was also shown, in patients with psoriatic arthritis in comparison to patients with normal psoriasis. Despite the presence of many works studying the level of cytokines in the course of the described diseases, there are no comparative publications for both groups of patients, taking into account the above cytokines. The effectiveness of anti-IL12/23 drugs in psoriatic arthritis is a confirmation of the obtained results of the studies. Additionally, the increased level of IL-17, both in patients with metabolic syndrome and with PsA, could indirectly indicate an increased cardiovascular risk in patients with affected joints in comparison to psoriasis affecting only the skin.

\section{Conclusions}

A lot of scientific research from recent years showed a connection between psoriasis and metabolic syndrome. Both nosological entities are accompanied by an increase in the level of proinflammatory cytokines, hyperuricemia, and abnormalities in excreted adipokines [7]. An accurate mechanism explaining the relation between the psoriasis and metabolic disorders is still not known, that is why further studies, evaluating the risk of co-incidence of the above entities, are necessary. The results of such works might lead to changes in therapy options and improve the results of psoriasis treatment.

\section{Acknowledgments}

The work was financed by statutory work fund no. 503-1137-4 of the Jagiellonian University Medical College.

\section{Conflict of interest}

The authors declare no conflict of interest.

\section{References}

1. Lowes MA, Suarez-Farinas M, Krueger JG. Immunology of psoriasis. Annu Rev Immunol 2014; 32: 227-55.

2. Gisondi P, Tessari G, Conti A, et al. Prevalence of metabolic syndrome in patients with psoriasis: a hospital-based casecontrol study. Br J Dermatol 2007; 157: 68-73.

3. Gönül M, Tatar I, Canpolat F, et al. Evaluation of abdominal fat index by ultrasonography and its relationship with psoriasis and metabolic syndrome. Adv Dermatol Allergol 2017; 34: 453-6. 
4. Işık S, Kılıç S, Öğretmen Z, et al. The correlation between the psoriasis area severity index and ischemia-modified albumin, mean platelet volume levels in patients with psoriasis. Adv Dermatol Allergol 2016; 33: 290-3.

5. Lakshmi S, Nath AK, Udayashankar C. Metabolic syndrome in patients with psoriasis: a comparative study. Indian Dermatol Online J 2014; 5: 132-7.

6. Nijsten T, Wakkee M. Complexity of the association between psoriasis and comorbidities. J Invest Dermatol 2009; 129: 1601-3.

7. Gerkowicz A, Pietrzak A, Szepietowski JC, et al. Biochemical markers of psoriasis as a metabolic disease. Folia Histochem Cytobiol 2012; 50: 155-70.

8. De Vecchis R, Baldi C, Palmisani L. Protective effects of methotrexate against ischemic cardiovascular disorders in patients treated for rheumatoid arthritis or psoriasis: novel therapeutic insights coming from a meta-analysis of the literature data. Anatol J Cardiol 2016; 16: 2-9.

9. Costa L, Caso F, Atteno M, et al. Impact of 24-month treatment with etanercept, adalimumab, or methotrexate on metabolic syndrome components in a cohort of 210 psoriatic arthritis patients. Clin Rheumatol 2014; 33: 833-9.

10. Chen DY, Chen YM, Hsieh TY, et al. Significant effects of biologic therapy on lipid profiles and insulin resistance in patients with rheumatoid arthritis. Arthritis Res Ther 2015; 17: 52.

11. Zuniga LA, Shen WJ, Joyce-Shaikh B, et al. IL-17 regulates adipogenesis, glucose homeostasis, and obesity. I Immuno 2010; 185: 6947-59.

12. Golden JB, McCormick TS, Ward NL. IL-17 in psoriasis: implications for therapy and cardiovascular co-morbidities. Cytokine 2013; 62: 195-201.

13. Di Cesare A, Di Meglio P, Nestle FO. The IL-23/Th17 axis in the immunopathogenesis of psoriasis. J Invest Dermatol 2009; 129: 1339-50.

14. Ait-Oufella H, Taleb S, Mallat Z, et al. Recent advances on the role of cytokines in atherosclerosis. Arterioscler Thromb Vasc Biol 2011; 31: 969-79.

15. Barnabe C, Martin BJ, Ghali WA. Systematic review and metaanalysis: anti-tumor necrosis factor alpha therapy and cardiovascular events in rheumatoid arthritis. Arthritis Care Res (Hoboken) 2011; 63: 522-9.

16. Jacobsson LT, Turesson C, Gulfe A, et al. Treatment with tumor necrosis factor blockers is associated with a lower incidence of first cardiovascular events in patients with rheumatoid arthritis. J Rheumatol 2005; 32: 1213-8.

17. Nguyen T, Wu JJ. Relationship between tumor necrosis factor-alpha inhibitors and cardiovascular disease in psoriasis: a review. Perm J 2014; 18: 49-54.

18. Popa C, van den Hoogen FHJ, Radstake TRDJ, et al. Modulation of lipoprotein plasma concentrations during long-term anti-TNF therapy in patients with active rheumatoid arthritis. Ann Rheum Dis 2007; 66: 1503-7.

19. Sinagra E, Perricone G, Romano C, et al. Heart failure and antitumor necrosis factor-alpha in systemic chronic inflammatory diseases. Eur J Intern Med 2013; 24: 385-3.

20. Hugh J, Van Voorhees AS, Nijhawan RI, et al. From the Medical Board of the National Psoriasis Foundation: the risk of cardiovascular disease in individuals with psoriasis and the potential impact of current therapies. I Am Acad Dermatol 2014; 70: 168-77.

21. Abdel-Hamid MF, Aly DG, Saad NE, et al. Serum levels of interleukin-8, tumor necrosis factor- $\alpha$ and gamma-interferon in Egyptian psoriatic patients and correlation with disease severity. J Dermatol 2011; 38: 442-6.

22. Anderson KS, Petersson S, Wong J, et al. Elevation of serum epidermal growth factor and interleukin 1 receptor antagonist in active psoriasis vulgaris. Br J Dermatol 2010; 163: 1085-9.

23. Takahashi H, Tsuji H, Hashimoto Y, et al. Serum cytokines and growth factor levels in Japanese patients with psoriasis. Clin Exp Dermatol 2010; 35: 645-9.

24. Kyriakou A, Patsatsi A, Vyzantiadis TA, et al. Serum levels of TNF-alpha, IL 12/23p40, and IL-17 in plaque psoriasis and their correlation with disease severity. J Immunol Res 2014; 2014: 467541.

25. Michalak-Stoma A, Bartosinska J, Kowal M, et al. Serum levels of selected Th17 and Th22 cytokines in psoriatic patients. Dis Markers 2013; 35: 625-31.

26. Arican O, Aral M, Sasmaz S, et al. Serum levels of TNF-alpha, IFN-gamma, IL-6, IL-8, IL-12, IL-17, and IL-18 in patients with active psoriasis and correlation with disease severity. Mediators Inflamm 2005; 2005: 273-9.

27. Zuniga LA, Shen WJ, Joyce-Shaikh B, et al. IL-17 regulates adipogenesis, glucose homeostasis, and obesity. J Immunol 2010; 185: 6947-59.

28. Alcorn N, Tierney A, Wu O, et al. Impact of anti-tumour necrosis factor therapy on the weight of patients with rheumatoid arthritis. Ann Rheum Dis 2010; 69: 1571.

29. Engvall IL, Tengstrand B, Brismar K, et al. Infliximab therapy increases body fat mass in early rheumatoid arthritis independently of changes in disease activity and levels of leptin and adiponectin: a randomised study over 21 months. Arthritis Res Ther 2010; 12: R197.

30. Briot K, Gossec L, Kolta S, et al. Prospective assessment of body weight, body composition, and bone density changes in patients with spondyloarthropathy receiving anti-tumor necrosis factor-alpha treatment. J Rheum 2008; 35: 855-61.

31. Giovanni T, Susan C, Carmine F, et al. Is serum Interleukin-17 associated with early atherosclerosis in obese patients? J Transl Med 2014; 12: 214.

32. Owczarczyk-Saczonek A, Placek W, Rybak-d'Obyrn J, et al. Influence of ustekinumab on body weight of patients with psoriasis: an initial report. Adv Dermatol Allergol 2014; 31: 29-31.

33. Pacholczyk M, Ferenc T, Kowalski J. The metabolic syndrome. Part I: Definitions and diagnostic criteria for its identification. Epidemiology and relationship with cardiovascular and type 2 diabetes risk. Postepy Hig Med Dosw 2008; 62: 530-42. 